\title{
O uso do Quantum Gis (QGIS) para caracterização e delimitação de área degrada por atividade de mineração de basalto no município de Tentente Portela (RS)
}

\author{
Gis Quantum usage (QGIS) to the characterization and delimitation of a degraded area by basalt mining \\ activity in the municipality of Tenente Portela (RS)
}

\author{
Natieli Luisa Torchetto';Rosemar de Queiroz'; Caroline Peyrot'; Eduardo Ruwer Patatt'; Carlos Henrique \\ Langner'; Leonardo Ochoa'; Ezequiel Koppe ${ }^{2}$
}

\author{
'Graduação em Engenharia Ambiental, Universidade Federal de Santa Maria, Frederico Westphalen - RS - Brasil \\ ${ }^{2}$ Professor Universitário, Universidade Federal do Ceará, Fortaleza - CE - Brasil
}

\section{Resumo}

A ascensão da construção civil desenvolveu um processo acelerado de extração de rochas, aumentando o numero e a extensão de pedreiras no Brasil. Essa atividade de extração de rochas causa diversas alterações no meio ambiente, sendo necessário o monitoramento dessas áreas degradadas através do uso do zoneamento ambiental. Para o monitoramento da mina de extração de Basalto do município de Tenente Portela (RS), utilizou-se do uso do software Quantum Gis (QGIS) para a caracterização da área, efetuando a delimitação da área da cava de extração e das regiões de depósito de rejeitos, as vias de acesso e pontos de interesse na área em questão. Para cada delimitação foram nomeados em pontos para melhor visualização das delimitações. O software Quantum Gis mostra-se, através das ferramentas ofertadas pelo mesmo para manipulação de dados espaciais, um bom método geotécnico para auxílio no planejamento de recuperação da área degrada, pois o software oferta mecanismos de levantamento de dados que facilitam o estudo do estado da área em questão.

Palavras-chave: Quantum Gis(QGIS); Monitoramento, Delimitação

\begin{abstract}
The rise of civil construction developed an accelerated process of extracting rock, increasing the number and extent of the quarries in Brazil . This activity of extracting rock causes several changes in the environment, monitoring of these degraded areas through the use of environmental zoning is required. To monitor the extraction of basalt from the municipality of Tenente Portela ( RS ) mine, we used Quantum GIS ( QGIS ) software to characterize the area, drafting the delimitation of the area of mining digging and regions of deposit tailings , access roads and points of interest in the area in. For each contour, it was nominated for best viewing points on the boundaries. The Quantum GIS software is shown, using the tools offered by them for handling spatial data, a good geotechnical method to aid in planning the recovery of degraded area because the software offer data collection mechanisms that facilitate the study of the state of area in question.
\end{abstract}

Keywords: Quantum GIS ( QGIS ) ; Monitoring, Delimitation 


\section{INTRODUÇÃO}

O subsolo brasileiro possui importantes depósitos minerais, onde partes dessas reservas são consideradas expressivas quando relacionadas mundialmente. O Brasil produz cerca de 70 substâncias, sendo 21 dos grupos de minerais metálicos, 45 dos não-metálicos e quatro dos energéticos (BARRETO, 2001), se enquadrando, segundo estudos do Departamento Nacional de Produção Mineral, DNPM, (2008), em um dos países com maior potencial mineral do mundo, juntamente com a Federação Russa, Estados Unidos, Canadá, China e Austrália.

As primeiras pedreiras que surgiram no Brasil tinham o processo de extração do basalto executado de forma simples, pois possuíam poucas tecnologias, sendo uma época que pode ser descrita com um desenvolvimento tecnológico tímido nas extrações de rochas, para o emprego na construção civil (GERMANI, 2002 citado em GEHLEN, 2008). Porem, segundo Bitar (1997), é uma atividade que causa alterações ambientais, como: supressão de áreas de vegetação, reconfiguração de superfícies topográficas, alteração de feições geomorfológicas e das encostas (instabilidade de taludes), impacto visual, aceleração de processos erosivos, aumento da turbidez e assoreamento de corpos d'água, emissão de gases e partículas no ar, ruídos, além da propagação de vibrações no solo.

O processo de regularização e legalização das pedreiras de basalto foi instituído em lei em 1990, pelas Fundações Estaduais de Proteção Ambiental, exigindo das empresas mineradoras o cumprimento da legislação ambiental, para obtenção das licenças inicial, prévia e de operação das jazidas (GEHLEN, 2008), onde a constituição Federal do Brasil de 1988, em seu Art. 225, Parágrafo $2^{\circ}$, impõe que aquele que explorar recursos minerais, deverá obrigatoriamente recuperar o ambiente degradado, de acordo com solução técnica exigida pelo órgão público competente, na forma da lei.

Para que seja atendido o processo de recuperação da área junto aos órgãos responsáveis pelas licenças de extração deverão ser adotadas medidas mitigadoras e de impacto ambiental obedecendo a um cronograma de desempenho dessas atividades como: etapas de recuperação, estabelecimento de objetivos a curto e a longo prazo, obras de engenharia na recuperação, manejo de solo orgânico, preparação do local para o plantio, seleção de espécies das plantas (GEHLEN, 2008).

Umas das formas de se garantir um controle dessas alterações adversas no meio ambiente é o uso do zoneamento ambiental, onde Barreto (2001) comenta que, o zoneamento ambiental é caracterizado como um dos instrumentos de gestão da Política Nacional do Meio Ambiente, sendo definido como um conjunto de procedimentos de natureza geoeconômica, voltados para a integração sistêmica e interdisciplinar da análise ambiental de um determinado local, objetivando disciplinar os distintos usos do solo e a consequente gestão racional e otimizada dos recursos naturais e ambientais da área objeto.

Desta forma, o Zoneamento Ambiental é um instrumento estratégico de planejamento e de localização de atividades degradadoras auxiliando na viabilização da inserção da variável ambiental em diferentes momentos do processo decisório, além de contribuir para a simplificação na elaboração do Estudo de Impacto Ambiental (EIA) e, consequentemente, do Licenciamento Ambiental de atividades (MONTAÑO et al., 2007).

\section{SISTEMA DE INFORMAÇÃO GEOGRÁFICA (SIG)}

A efetivação de um Sistema de Informações Geográficas em um computador está condicionada a qualidade da transposição de formas e objetos do mundo real e suas interações.

A partir do geoprocessamento se tem a relação de um dado espacial com o modelo de dados, onde o dado espacial retrata representações do mundo real em uma determinada escala e essas representações tem forma, cores e localização.

O modelo de dados mais utilizado para representar uma entidade é por um par de dados: localização geográfica e atributo. O atributo expõe as características da entidade, sendo descrito em termos quantitativos ou qualitativos, conforme uma escala nominal, ordinal, intervalo ou razão. E a entidade representada pode ter vários atributos que a caracterizam (MIRANDA, 2010).

O universo a ser modelado para aplicações de geoprocessamento integram os mais diferentes tipos de dados provenientes de diversas fontes como mapas, imagens de satélites, cadastros (FREITAS, 2001).

O estudo dos fenômenos espaciais é abstraído em três classes: ponto, linha e áreas ou polígonos, 
sendo que o critério que os diferencia é a dimensionalidade. A dimensão do ponto é zero, da linha é um, da área (polígono) é duas e a superfície é representada por três dimensões. (MIRANDA, 2010).

Nesse contexto, as geotecnologias podem ser grandes aliadas aos estudos ambientais, pois ofertam ferramentas que subsidiam diversas demandas do planejamento e gestão territorial (MORAES, 2010). Ferramentas que abrangem análise espacial de ambientes, permitindo o conhecimento do uso do solo, área, comprimento, entre outros. Ocasionando, assim, uma maior capacidade de avaliação, gerenciamento e planejamento de áreas passíveis de degradação, poluição e ocupação indevida.

Sendo, portanto, a aplicação de Sistemas de Informações Geográficas (SIG), e outras geotecnologias (sensoriamento remoto, GPS, geoprocessamento), um fator determinante no zoneamento ambiental, planejamento e gestão territorial.

\section{I Sobre o Quantum Gis (QGIS)}

Entre as diversas geotecnologias disponíveis no mercado, para manipulação de dados espaciais, uma delas é o software Quantum Gis (QGIS).

O Quantum Gis é um software gratuito, licenciado pela GNU (General Public License), baseado em um SIG (Sistema de Informação Geográfica).

A interface do software é simples e de fácil manuseio, o programa oferta várias ferramentas que possibilitam visualizar, gerenciar, editar, analisar os dados e compor mapas impressos, obter impressão com determinadas screenshots e uma lista de recursos mais detalhada.

Permitindo, segundo Almeida (2011), consultas espaciais, exploração interativa de dados, identificação e seleção de geometrias, pesquisa, visualização e seleção de atributos e criação de simbologia vetorial e raster. Suportando as bases de dados geográficas PostGIS, SpatiaLite e SQL Anywhere, além de todos os formatos suportados pela biblioteca GDAL (Geospatial Data Abstraction Library) (ALMEIDA, 2011).

\section{OBJETIVO}

Realizar através do uso do software Quantum Gis a caracterização da área da mina de extração de Basalto do município de Tenente Portela (RS), efetuando a delimitação da área da cava de extração e das regiões de depósito de rejeitos, as vias de acesso e pontos de interesse na área em questão.

\section{METODOLOGIA}

\section{I Caracterização do município}

O município de Tenente Portela está situado no Estado de Rio Grande do Sul, na porção norte do Estado, como mostrado na figura 1, na região do Alto Uruguai, possui uma área de $338,083 \mathrm{~km}^{2}$, uma altitude média de $390 \mathrm{~m}$, conforme senso demográfico de 2010 , com 13.719 habitantes. As coordenadas geográficas do município são: $27^{\circ} 22^{\prime} 15^{\prime \prime}$ sul e a uma longitude $53^{\circ} 45^{\prime} 28^{\prime \prime}$ oeste.

Tem como limites ao Norte os municípios de Derrubadas e Barra do Guarita, ao sul com Miraguaí, ao Leste com Palmitinho, Vista Gaúcha e Erval Seco e ao Oeste com o município de Três Passos.

A economia do município baseia-se na agricultura, tendo como principais culturas cultivadas a soja, o milho e o trigo, sendo também de grande importância econômica as atividades: leiteira, suinocultura e avicultura.

A região noroeste do estado (RS), conforme Breuniget al. (2011), apresentam clima do tipo Cfa (classificação de Köppen), denominado subtropical, com temperaturas médias do mês mais quente (janeiro) superiores a $22^{\circ} \mathrm{C}$ e no mês mais frio (julho) oscilando entre $-3^{\circ} \mathrm{C}$ e $18^{\circ} \mathrm{C}$. A precipitação anual é de aproximadamente $1.665 \mathrm{~mm}$, bem distribuída ao longo do ano.

\subsection{Caracterização da mina de extração de basalto através do Quantum Gis}

A execução da delimitação da área iniciou-se com a busca do município de Tenente Portela através do complemento open layers plugin (add google hybrid layer), após realizou-se a aproximação para a região onde localiza-se a pedreira.

Em seguida adicionaram-se três camadas do tipo shape. Sendo a primeira camada vetorial 


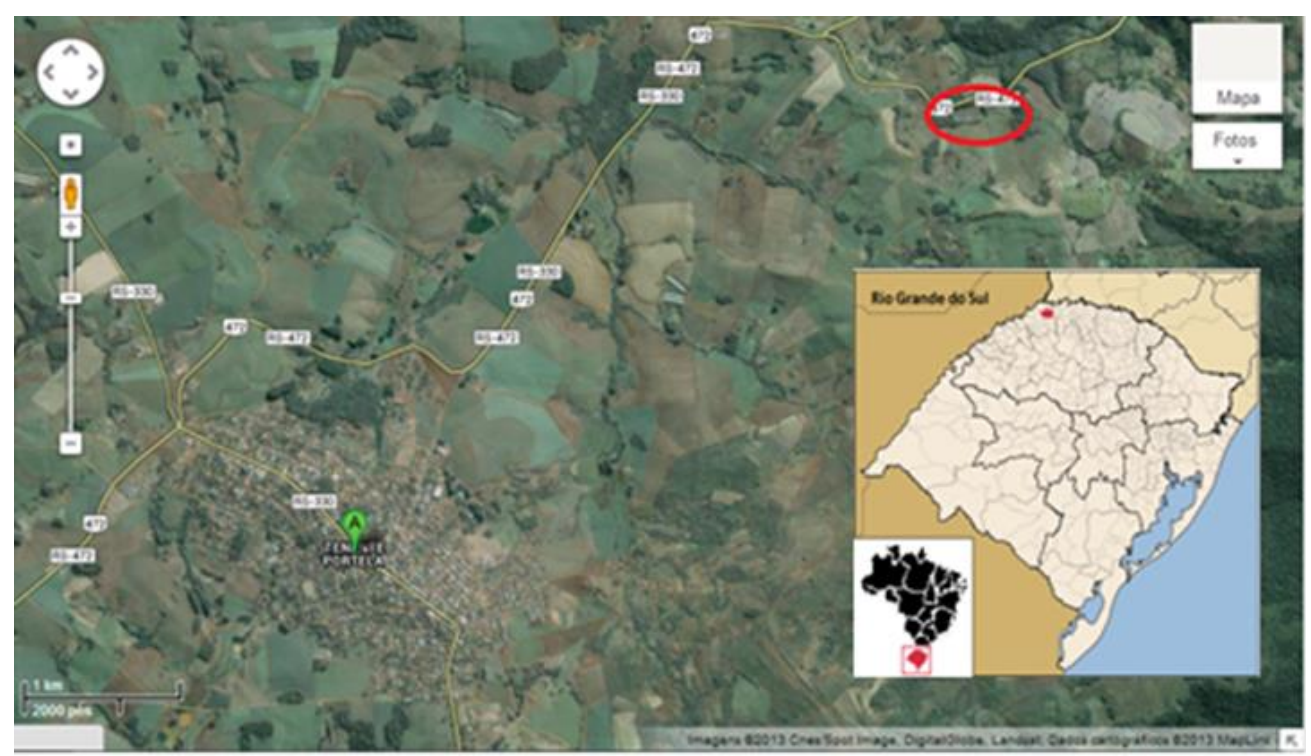

Figura 1: Localização de Tenente Portela.

Fonte: Google Earth, 2013 editada por Autores.

do tipo ponto destinada à identificação de pontos de interesse na área de estudo, a segunda camada vetorial foi do tipo linha para delimitação das vias de acesso a área de estudo e a terceira camada vetorial foi do tipo polígono para delimitação das regiões de interesse do estudo, abrangendo cava de extração, zonas de depósito de rejeitos da atividade e região total de atuação de atividade degradante. Para todas as três camadas do tipo shape adicionadas o Sistema de Coordenadas de Referência (SCR) selecionado foi o Córrego Alegre (UTM zone 22s).

Para cada camada vetorial adicionada realizou-se a edição das características das mesmas, através da tabela de atributos, a fim de identificar o que cada dado representa do espaço real, bem como calcular suas distancias e/ou área de abrangência.

\section{RESULTADOS E DISCUSSÕES}

Através do uso das ferramentas ofertadas pelo software QGIS foi possível identificar a área de interesse do estudo, originando a sua localização e distância da cede urbana do município, estando à mesma há 4791,7, como demonstrado na Figura 1. Com o auxílio do Google Eart, foi realizada a localização de área de estudo a nível estadual e nacional.

Após a localização da área procedeu-se a marcação dos pontos 1, 2, 3 e 4 que correspondem a maquinários, galpão de equipamentos 1, galpão de equipamentos 2 e moradia, respectivamente. Como explanado na Figura 2 que conta, além da identificação descrita acima, com o par de coordenadas geográficas de cada ponto na área de estudo.

Posteriormente efetuou-se a demarcação das vias de acesso a mina de extração de basalto como demonstra a Figura 3, sendo a via 1 a estrada para a parte superior da cava de extração com aproximadamente 514, 83 metros (m) de extensão, a via 2 representa a estrada de acesso a parte do maquinário da pedreira contando com 102,51 m e a via 3 corresponde a estrada na cava de extração com $183 \mathrm{~m}$.

Depois se realizou a identificação das áreas de interesse do estudo, como exemplificado na Figura 4, sendo a região 1 a cava de extração de basalto com aproximadamente 1,908 hectare (ha) de extensão, região 2 corresponde a área de depósito de rejeito 1 contando com 0,1646 ha, a região 3 demonstra a área de depósito de rejeito $2 \operatorname{com} 0,101$ ha e a região 4 corresponde a área total de atuação da pedreira abrangendo aproximadamente 8,359 ha. 


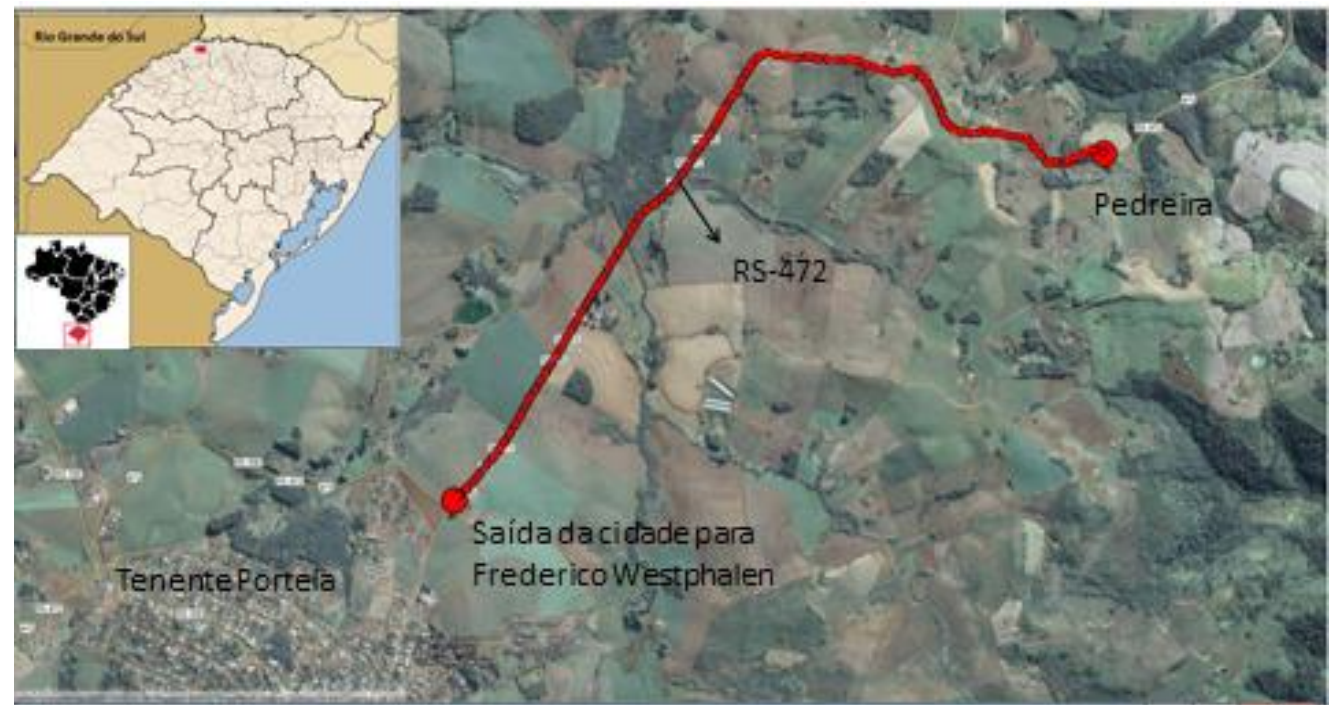

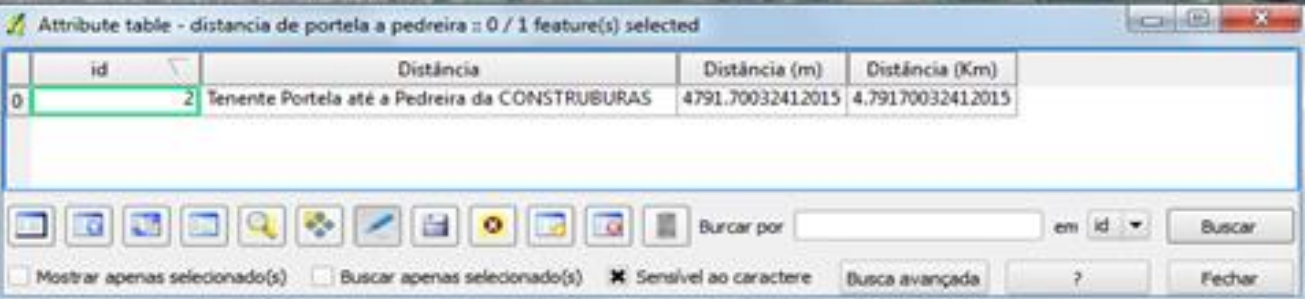

Figura 1: Localização da mina de exploração de basalto de Tenente Portela (RS)

Fonte: QGIS

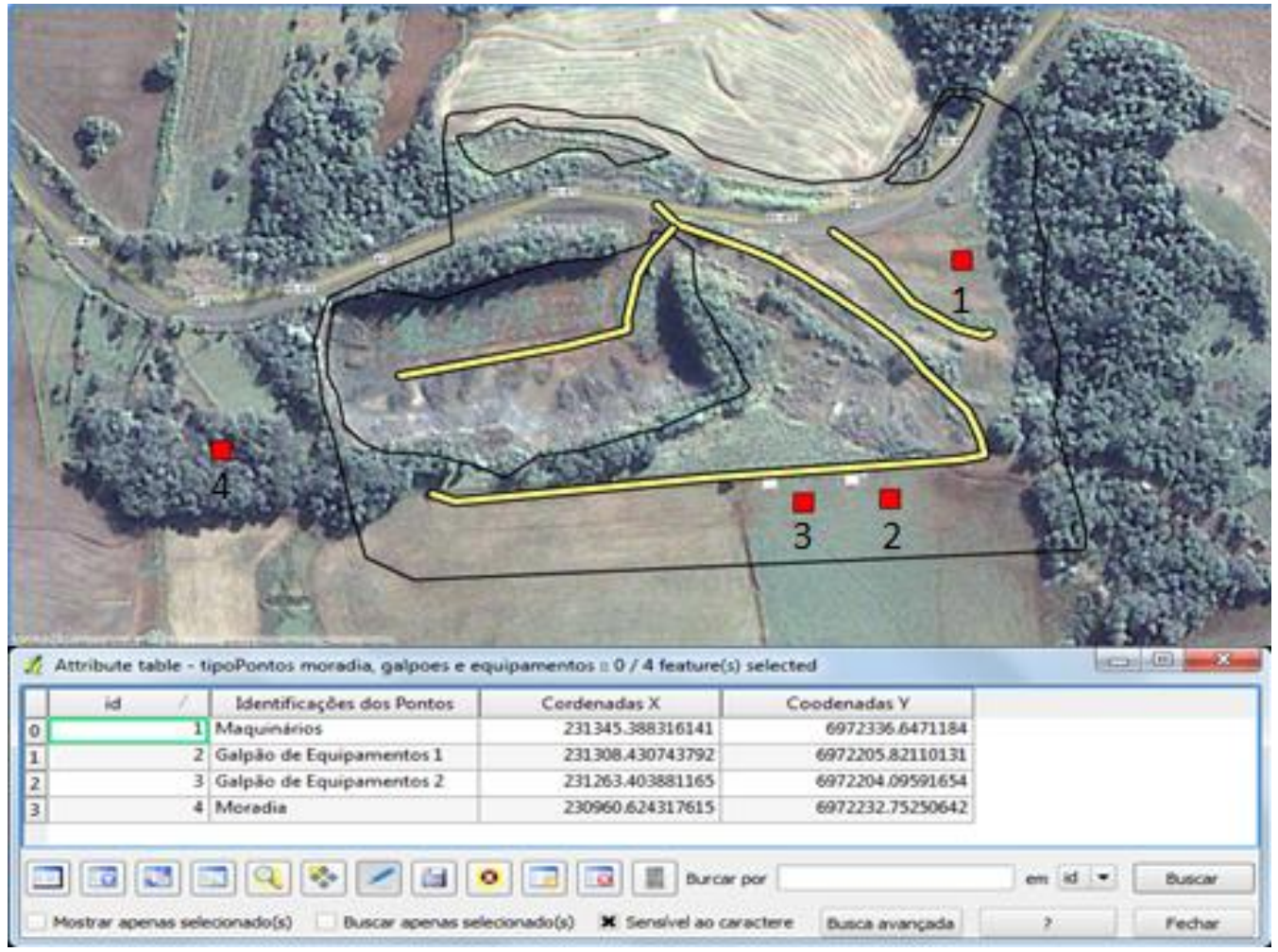

Figura 2: Equipamentos dispostos na área de estudo

Fonte: QGIS 


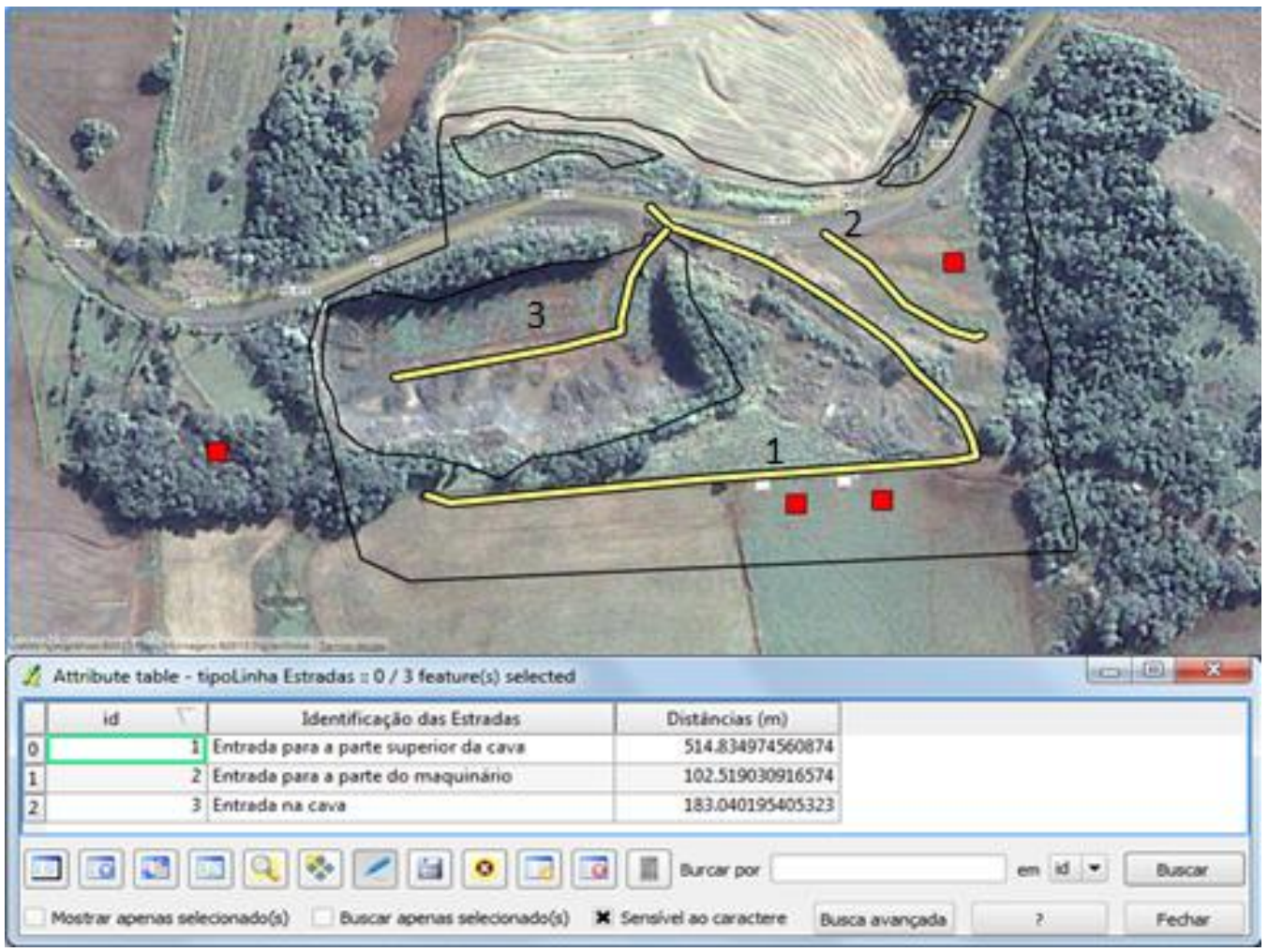

Figura 3: Vias de acesso a mina de exploração de basalto de Tenente Portela (RS)

Fonte: QGIS

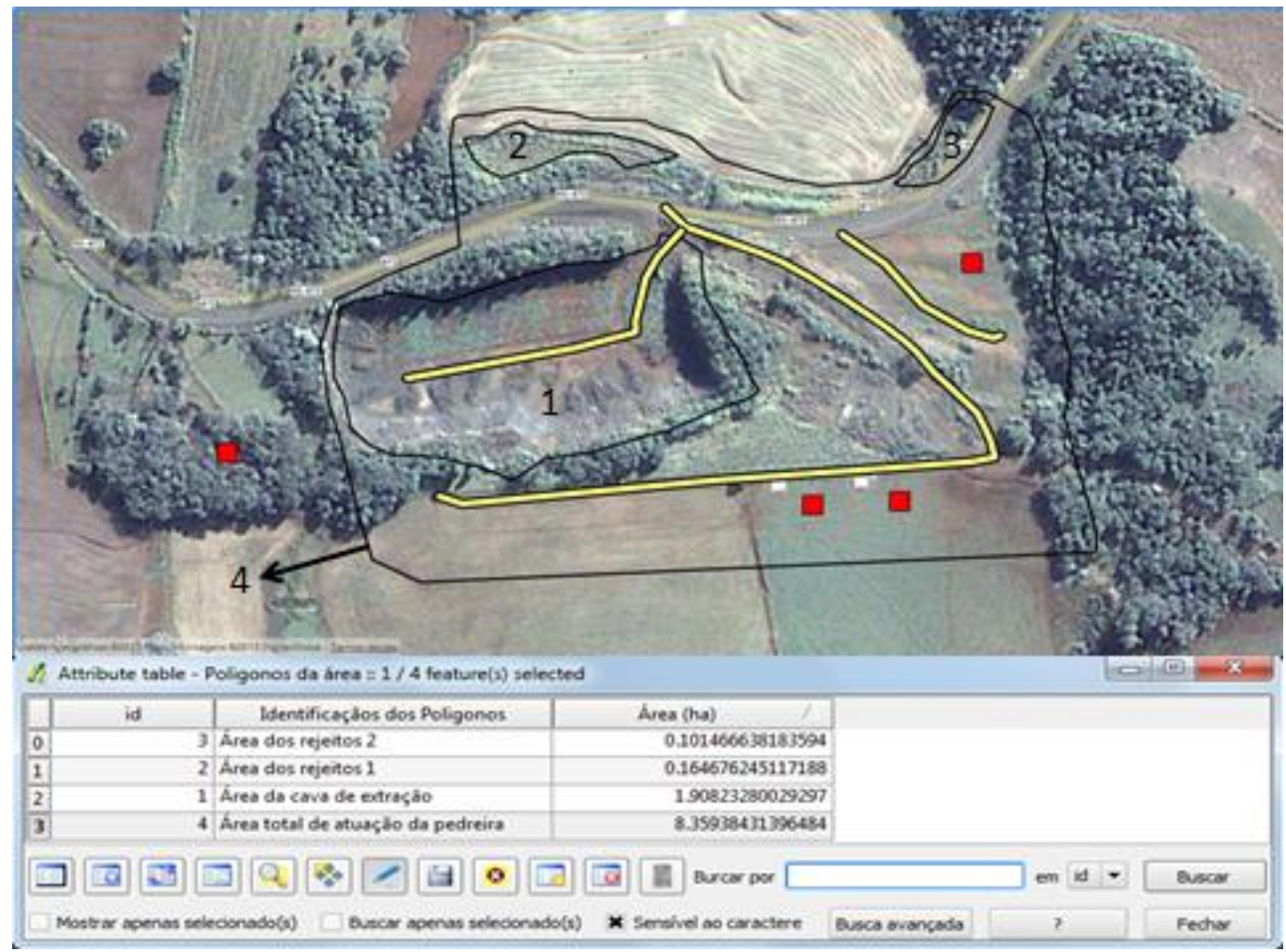

Figura 4: Delimitação das áreas de interesse do estudo

Fonte: QGIS 


\section{CONCLUSÃO}

Tendo em vista que o processo de extração de basalto na mina do município de Tenente Portela (RS) é uma atividade degradante do meio ambiente, se faz necessário o estudo de um plano de recuperação para a área degrada.

O software Quantum Gis mostrou-se, através das ferramentas ofertadas pelo mesmo para manipulação de dados espaciais, um bom método geotécnico para auxílio no planejamento de recuperação de uma área degrada. Pois o software oferta mecanismos de levantamento de dados que facilitam o estudo do estado da área em questão, por meio de sua identificação, localização, a extensão da degradação entre outros, e consequentemente prestando assistência ao planejamento, gestão territorial e zoneamento ambiental.

\section{REFERÊNCIAS}

AUMOND, J. J.; Adoção de uma nova abordagem para a recuperação de área degradada pela mineração. Florianópolis, 2007.Tese (Doutorado), Universidade Federal de Santa Catarina.

ALMEIDA, R. P. O.; SÁNCHEZ, L. E.; Revegetação de áreas de mineração: critérios de monitoramento e avaliação do desempenho.R. Árvore, Viçosa-MG, v.29, n.1, p.47-54, 2005.

BAIÃO, Joaquim O. Plano ambiental e de recuperação paisagística. Losoambiente: serviços e projetos ambientais Ltda. 2011. Disponível em:<http://www.google.com.br/url?sa=t\&rct=j\&q=tecnica $\% 20$ de $\% 20$ interven $\%$ C3\%A7ao\%20de $\% 20$ renivelamento $\% 20 \&$ source $=$ web $\& \mathrm{~cd}=1 \& \mathrm{cad}=$ rja $\&$ ved $=0 \mathrm{CCwQ}$ FjAA\&url=http\%3A\%2F\%2Fwww.ccdr-a.gov.pt\%2Fexplorers\%2Fpedreirabendada\%2FDefault.asp\% 3Fprecommand\%3DDownload\%26folder\%3D\%26file\%3DPARP.pdf\&ei=SyPOUZaJGMHm0QGB_ oF4\&usg=AFQjCNEHZxbsxf5-sjx05HEaL1glXwuqDw\&bvm=bv.48572450,d.dmQ>. Acesso: 28 jun. 2013.

BARRETO, M. L. Mineração e desenvolvimento sustentável: desafios para oBrasil. Rio de Janeiro: CETEM/ MCT, 2001. 215p.

BREUNIG, Fábio M. et al. Caracterização espectral e temporal da vegetação nativa do Parque Estadual do Turvo e da Terra Indígena do Guarita - RS, com produtos MODIS. SBSR:2011. Disponível em:<http://www. dsr.inpe.br/sbsr2011/files/p0272.pdf>. Acesso em: 29 jun. 2013.

Constituição da República Federativa do Brasil de 1988. Site oficial. Disponível em: <http://www.jusbrasil. com.br/legislacao/1033700/constituicao-da-republica-federativa-do-brasil-1988\#art225>. Acessado em: 25 jun. 2013.

DNPM - Departamento Nacional de Proteção Mineral. Site oficial. Disponível em:<http://www.dnpm.gov. br/>. Acessado em: 25 jun. 2013.

DNPM- Departamento Nacional De Produção Mineral. Legislação Mineral. Disponível em: http://www. dnpm.gov.br/conteudo.asp?IDSecao=67. Acesso: 29 jun. 2013.

FREITAS, Cinthia Obladen de Almendra. sig para diagnóstico e monitoramento ambiental da área degradada pela mineração de xisto em São Mateus do Sul - PR. ABES, 2001. Disponível em:< http://www.bvsde.paho. org/bvsacd/abes97/mateus.pdf>. Acesso em: 16 jul. 2013.

GEHLEN, I. V. Exploração de basalto na região das missões do Estado do Rio Grande do Sul. No. 23 - 2008.

MANUAL DE MEIO AMBIENTE. Proposta para o licenciamento ambiental de pedreiras e jazidas: anexo 4. Versão 1,13 out. 2000.

MIRANDA, José I. Fundamentos de Sistemas de Informações Geográficas. -2. ed. rev. atual. - Brasília, DF : 
Embrapa Informação Tecnológica, 2010.

MONTAÑO, M., OLIVEIRA, I. S. D., RANIERI, V. E. L., FONTES, A. T., SOUZA, M. P. (2007). O Zoneamento Ambiental e a sua Importância para a Localização de Atividades. Pesquisa e Desenvolvimento Engenharia de Produção No. 6, p. 49- 64.

MORAES, Iranilda Silva et al. A utilização do sig como ferramenta para indicação de áreas possíveis a implantação de aterro sanitário na região metropolitana de Belém - PA. Recife, 2010. Disponivel me:< http:// www.ufpe.br/cgtg/SIMGEOIII/IIISIMGEO_CD/artigos/CartografiaeSIG/SIG/R_215.pdf>. Acesso em 15 jul. 2013. 